\title{
The prevalence of rabies cases in the territory of Azerbaijan, January 2015-June 2016
}

\author{
Nigar Safi ${ }^{\star}$, Kliment Asadov, Shalala K. Zeynalova, Eldar Gasanov and Natig Javadov
}

State Veterinary Control Service, Baku, Azerbaijan

\section{Objective}

to show the instability of an epizootic situation on rabies cases of animals in the Republic of Azerbaijan, on the example of the cases analysis in Electronic Integrated Disease Surveillance System (EIDSS) electronic reporting system

\section{Introduction}

Rabies is an infectious disease which was and remains to be one of the most serious diseases of all species of hematothermal animals and humans, in many regions of the world. The epizootic situation on rabies in the Republic of Azerbaijan has been unfavorable for many years, which is confirmed by scientific data and the veterinary cases reporting in the EIDSS system. This system was introduced in the country in 2009 and is the electronic System of disease control. The program allows to provide monitoring and prevention of diseases within the concept "One World - One Health System" by integration of systems of observation of animal diseases, human diseases, and disease carriers.

\section{Methods}

On the basis of the data on rabies cases entered in special forms and also aggregative data collected on anti-rabies vaccination, the analysis of information on quantity of cases and their prevalence on administrative and territorial units (rayons) of the country is carried out. The graphical analysis (charts and the map) on the basis of necessary criteria are constituted in the analyses module, visualization of the AVR reporting and in the Microsoft Excel program.

\section{Results}

The analysis of the rabies cases confirmed at the Virology department of the Republican Veterinary Laboratory shows that rabies has been identified in 36 cases in 2015, 25 cases in January - June, 2016, in total 61 cases has been registered for the period of "January 2015 - June 2016". An epizootologically unfavorable situation is revealed in 27 regions. The most unfavorable situation is the northwest regions of the country, the most part of which is covered with mountainy-forest area with domination of wild fauna. Specific structure of animals: dogs -31 cases in 19 areas $(51 \%)$, cattle -21 cases in 12 areas $(34 \%)$, a small cattle- 1 case $(2 \%)$, wild animals (specify types) -8 cases in 8 areas (13\%) that is visually shown on charts 1 and 2 . The cattle were bitten by wolves and jackals.

\section{Conclusions}

Thus, prevalence of rabies cases of different species of animals in the country, once again proves natural and focal character of the disease: the reservoir of rabies is in the wild nature and geographical conditions impact the spread of rabies.

Cases of rabies in animals are registered annually. In 2015, vaccination captured about 250000 dogs, and 244400 dogs were vaccinated in the first 6 months of 2016. Despite a huge group of vaccinations, restriction of rabies spread isn't observed and the tendency is trending to the increase of rabies case indicators amongst the dogs.

It is necessary to pay close attention to preventive vaccination of domestic (including non-productive) animals. If materiel resources are available, it is possible to carry out the vaccination of the cattle in the territories adjacent to the forests. In the threatened territories with woodlands, there is no alternative to oral vaccinations, which is confirmed by positive experience of many countries. There is an extreme need of carrying out of oral vaccination of wild carnivorous animals with obligatory control of the immune status.

Keywords

rabies; detection; Electronic reporting; vaccination

\section{*Nigar Safi}

E-mail: hasanovag@state.gov 